\title{
CRIANÇAS COM PNEUMONIA ATENDIDAS PELA FISIOTERAPIA NA ENFERMARIA
}

\author{
CHILDREN WITH PNEUMONIA ASSISTED BY \\ PHYSICAL THERAPY IN A HOSPITAL WARD
}

Ana Elisa Luiz Marques (ORCID: 0000-0001-8000-8392) Natália Maria Finato (ORCID: 0000-0002-8997-3460) ${ }^{1,2}$ Alexandre Lins Werneck (ORCID: 0000-0002-2911-8091) 2,

Autor correspondente: Ana Elisa Luiz Marques e-mail: ana.elisa.marques@hotmail. com

\footnotetext{
${ }^{1}$ Hospital da Criança e Maternidade de São José do Rio Preto - SP.

${ }^{2}$ Programa de Pós-Graduação Stricto Sensu em Enfermagem. Faculdade de Medicina de São José do Rio Preto.

${ }^{3}$ Comissão de Aprimoramento e Aperfeiçoamento da Faculdade de Medicina de São José do Rio Preto
}

Trabalho de conclusão de curso de Ana Elisa Luiz Marques intitulado "Crianças com pneumonia atendidas pela fisioterapia na enfermaria" apresentado ao programa de pós-graduação em fisioterapia respiratória infantil da Faculdade de medicina de São José do Rio Preto - SP.

\section{RESUMO}

Objetivo: Identificar as características de crianças com pneumonia atendidas pela equipe de fisioterapia na enfermaria de um hospital universitário com o intuito de nortear o implemento de protocolos de atendimento direcionados a essa população. Métodos: Estudo de corte transversal com delineamento descritivo e abordagem quantitativa do tipo analítica com correlação entre variáveis. Os dados coletados foram sexo, faixa etária, antecedentes pessoais, complicações pulmonares, passagem pela UTI e necessidade de suporte ventilatório. Resultados: Nesta pesquisa, 62 crianças foram incluídas, $61,3 \%$ eram do sexo masculino. A faixa etária entre 29 dias e 2 anos teve $54,8 \%$ do total, $11,5 \%$ apresentavam alguma síndrome genética, e 18,0\%, doenças neurológicas. Além disso, 21,0\% apresentaram derrame pleural, $20,3 \%$ necessitaram de suporte em unidade de terapia intensiva. O percentual de crianças que utilizou a oxigenoterapia foi de $24,2 \%$. Conclusão: Houve predomínio do sexo masculino, com a faixa etária entre 29 dias e dois anos, parte das crianças era traqueostomizada ou tinha como antecedentes pessoais doenças neurológicas ou síndromes genéticas. A fisioterapia para crianças com pneumonia é controversa, de modo que esse conhecimento possibilitará nortear protocolos que proporcionem uma indicação mais criteriosa e uma melhor assistência.

Palavras-chave: Epidemiologia; Criança; Pneumonia; Fisioterapia.

\begin{abstract}
Objective: To identify the characteristics of children with pneumonia assisted by the physical therapy team in the ward of a university hospital in order to guide the implementation of care protocols for this population. Methods: Cross-sectional study with descriptive design and quantitative approach of analytical type with correlation between variables. The data collected were gender, age range, personal antecedents, pulmonary complications, intensive care unit visit, and ventilatory support needs. Results: In this study, 62 children were included, $61.3 \%$ were male. The age group between 29 days and 2 years was $54.8 \%$ of the total, $11.5 \%$ had some genetic syndrome, and $18.0 \%$ had neurological diseases. $21.0 \%$ had pleural effusion, $20.3 \%$ needed intensive care unit support. The percentage of children who used oxygen therapy was $24.2 \%$. Conclusion: There was a predominance of males, with ages ranging from 29 days to two years, part of the children were tracheostomized or had as personal background neurological diseases or genetic syndromes. Physical therapy for children with pneumonia is controversial, so this knowledge will make it possible to guide protocols that provide a more careful indication and better assistance.
\end{abstract}

Keywords: Epidemiology; Child; Pneumonia; Physical Therapy Specialty. 


\section{INTRODUÇÃO}

Crianças apresentam um maior risco para complicações no trato respiratório, visto que possuem particularidades anatômicas e fisiológicas que favorecem a obstrução e o colapso das vias respiratórias ${ }^{1}$. A pneumonia é uma infecção associada a uma inflamação da parte inferior do trato respiratório, representada por consolidação alveolar ou infiltração do tecido intersticial por células inflamatórias, o que ocasiona prejuízo na mecânica respiratória ${ }^{2}$. Os agentes causadores mais comuns são bactérias e vírus, entre eles, o Streptococcus pneumoniae, o Haemophilus influenza e o vírus sincicial respiratório ${ }^{3}$.

A taxa de mortalidade infantil mundial, que inclui a mortalidade por pneumonia, diminuiu consideravelmente desde 2000. Entretanto, ainda permanece alta, pois estima-se que 921 mil crianças com menos de 5 anos de idade morreram vítimas de pneumonia em $2015^{4-5}$. Entre todas as doenças que acometem as crianças menores, ela representa uma significativa parcela, portanto, os esforços para a melhoria da saúde da criança envolvem prioritariamente o combate a essa doença ${ }^{6}$.

A desnutrição foi identificada como fator de risco para a mortalidade de crianças menores de 5 anos por infecção da parte inferior do trato respiratório, que, somada à poluição do ar, tanto no ambiente doméstico quanto ambiental, e ao uso frequente de antibióticos, integra-se como alvo de medidas de intervenção no combate mundial da doença ${ }^{7}$. Outros fatores também são descritos, como prematuridade, baixo peso ao nascer, ausência de aleitamento materno, outras doenças de base, doenças pulmonares e cardiológicas, necessidade de ventilação mecânica, uso de sonda ou de cânula nasogástrica, macro ou microaspiração de secreção traqueobrônquica e vacinação incompleta ${ }^{8}$.

A fisioterapia respiratória consiste em um tratamento que utiliza intervenções mecânicas, fundamentadas na fisiologia respiratória ${ }^{9}$. Pode facilitar a troca gasosa, promover a expulsão das secreções pulmonares e, assim, ocasionar a diminuição do trabalho respiratório, sendo rotineiramente prescrita como parte do tratamento da pneumonia ${ }^{10}$.

A fisioterapia respiratória compreende o uso de variadas técnicas manuais de percussão, vibração e compressão torácica, estímulo e assistência à tosse, exercícios respiratórios e posicionamento. Desempenha papel significativo na eliminação de secreções das vias respiratórias e na expansão pulmonar, assim como na melhora da eficiência dos músculos respiratórios 9 .

A instituição, o paciente e o profissional de saúde encontram benefícios a partir do conhecimento do perfil dos indivíduos internados em uma enfermaria ${ }^{11}$. Existem poucos trabalhos com foco na caracterização de crianças com pneumonia, com prescrição para fisioterapia em enfermaria pediátrica. Dentro desse contexto, a presente pesquisa teve como objetivo identificar as características de crianças com pneumonia atendidas pela equipe de fisioterapia, na enfermaria de um hospital universitário, com o intuito de nortear o implemento de protocolos de atendimento, direcionados a essa população.

\section{MÉTODOS}

Esta pesquisa foi aprovada pelo Comitê de Ética em Pesquisa da Faculdade de Medicina de São José do Rio Preto, sob o parecer $n^{\circ}$ 3.641.627. Trata-se de um estudo transversal com delineamento descritivo e abordagem quantitativa do tipo analítica com correlação entre variáveis, cuja população foi composta por prontuários de crianças com diagnóstico de pneumonia e com prescrição para fisioterapia, internadas no setor da Enfermaria Pediátrica de um hospital universitário. 
A seleção foi feita por meio da amostragem não probabilística em sequência, ou seja, envolveu a pesquisa dos prontuários acessíveis em um intervalo de tempo específico (outubro, novembro e dezembro de 2019), sem tamanho de amostra especificado. As crianças com prescrição médica, para o atendimento fisioterapêutico, foram identificadas pelos pesquisadores mediante consulta a um censo, gerado diariamente pelo sistema operativo institucional. Dessa forma, os prontuários puderam ser identificados e incluídos no estudo a partir da confirmação do diagnóstico de pneumonia. Foram analisados 62 prontuários, excluídos os que não tiveram prescrição médica para fisioterapia e cujo diagnóstico não era de pneumonia. As variáveis sociodemográficas coletadas foram sexo, faixa etária, antecedentes pessoais, complicações pulmonares, passagem pela UTI e necessidade de suporte ventilatório.

Os dados foram coletados diariamente nos prontuários eletrônicos dos pacientes identificados, no período da tarde, e transcritos na ficha padronizada, durante os meses de duração da pesquisa. Após a tabulação dos dados coletados, foram exercidas duas funções de análises estatísticas: descritiva e inferencial. De maneira descritiva, foi traçado o perfil da amostra estudada, contemplando as variáveis analisadas e seus desdobramentos. Os dados foram replicados de forma absoluta e relativa nessa primeira parte. No âmbito inferencial, como objetivo estatístico, foi traçada a análise de dependência e predição entre as variáveis propostas no escopo do trabalho. Outrossim, foi executado o teste de normalidade de Kolmogorov Smirnov. Para análise das variáveis paramétricas, utilizou-se, dentro dos padrões esperados, o teste de correlação de Pearson. Os resultados da dependência entre as variáveis propostas foram obtidos por meio de análise entre os valores de $\mathrm{P}$ (significância). Todas as análises foram obtidas por intermédio do software SPSS Statistics ${ }^{\circledR}$ (versão 23), atrelado às funcionalidades da ferramenta Microsoft Excel ${ }^{\circledR}$ (versão 2016). Para descrição dos resultados, foram calculadas as frequências relativas e absolutas, média, desvio-padrão e mediana. Para conhecer os desfechos das variáveis não paramétricas, foi aplicado o teste U de Mann-Whitney. O resultado foi considerado significativo quando $\mathrm{p}<0,05$.

\section{RESULTADOS}

Marques et al

Os dados das 62 crianças incluídas neste

estudo estão apresentados na tabela 1 .

Tabela 1. Distribuição das crianças e respectivas porcentagens em relação aos dados coletados. São José do Rio Preto, SP, Brasil, 2020

\begin{tabular}{|c|c|c|}
\hline Variáveis & $\mathrm{N}$ & $\%$ \\
\hline \multicolumn{3}{|l|}{ Sexo } \\
\hline Feminino & 24 & $38,7 \%$ \\
\hline Masculino & 38 & $61,3 \%$ \\
\hline \multicolumn{3}{|l|}{ Faixa etária } \\
\hline Neonatal (0 a 28 dias) & 0 & $0,0 \%$ \\
\hline Lactente ( 29 dias a 2 anos) & 34 & $54,8 \%$ \\
\hline Pré-escolar ( 3 a 6 anos) & 17 & $27,4 \%$ \\
\hline Escolar (7 a 11 anos) & 9 & $14,5 \%$ \\
\hline$>11$ anos & 2 & $3,2 \%$ \\
\hline \multicolumn{3}{|l|}{ Antecedentes Pessoais } \\
\hline Crianças Hígidas & 36 & $59,0 \%$ \\
\hline Problemas respiratórios & 4 & $6,6 \%$ \\
\hline Síndrome genética & 7 & $11,5 \%$ \\
\hline Problemas neurológicos & 11 & $18,0 \%$ \\
\hline Problemas cardiocirculatórios & 3 & $4,9 \%$ \\
\hline \multicolumn{3}{|l|}{ Traqueostomia } \\
\hline Não & 52 & $83,9 \%$ \\
\hline Sim & 10 & $16,1 \%$ \\
\hline \multicolumn{3}{|l|}{ Complicaçöes } \\
\hline Não houve & 45 & $72,6 \%$ \\
\hline Derrame pleural & 13 & $21,0 \%$ \\
\hline Pneumatocele & 0 & $0,0 \%$ \\
\hline Atelectasia & 4 & $6,5 \%$ \\
\hline Outras & 0 & $0,0 \%$ \\
\hline \multicolumn{3}{|l|}{ Dreno de tórax } \\
\hline Não & 58 & $93,5 \%$ \\
\hline Sim & 4 & $6,5 \%$ \\
\hline \multicolumn{3}{|l|}{ Passagem pela UTI } \\
\hline Não há & 47 & $79,7 \%$ \\
\hline Sim & 12 & $20,3 \%$ \\
\hline \multicolumn{3}{|l|}{ Ventilação mecânica } \\
\hline Não necessitou & 57 & $96,6 \%$ \\
\hline Ventilação mecânica & 1 & $1,7 \%$ \\
\hline Ventilação não-invasiva & 1 & $1,7 \%$ \\
\hline \multicolumn{3}{|l|}{ Oxigenioterapia } \\
\hline Não & 47 & $75,8 \%$ \\
\hline Sim & 15 & $24,2 \%$ \\
\hline \multicolumn{3}{|l|}{ Interface utilizada } \\
\hline Nenhuma & 47 & $75,8 \%$ \\
\hline Cateter nasal & 12 & $19,4 \%$ \\
\hline Máscara de venturi & 1 & $1,6 \%$ \\
\hline Máscara de reservatório & 2 & $3,2 \%$ \\
\hline
\end{tabular}




\section{DISCUSSÃO}

Identificar as características dos indivíduos hospitalizados em uma unidade possibilita predizer recursos, coordenar processos, bem como permite o treinamento do pessoal, de maneira a propiciar uma assistência mais adequada a esses pacientes ${ }^{12}$. A pneumonia é a doença mais prevalente entre as solicitações de atendimento fisioterapêutico no hospital. Assim, iniciativas que permitam melhorar a assistência a essa população são relevantes.

Com relação ao sexo, em nossa pesquisa, observou-se que $61,3 \%(n=38)$ das crianças eram meninos. Estudos relataram o predomínio do sexo masculino em doenças respiratórias na infância com relação às taxas de morbidade e hospitalização ${ }^{13}$. No Brasil, fatores socioculturais, associados a uma maior liberdade para prática de esportes, estão ligados a uma grande exposição do sexo masculino a agentes infecciosos, por isso os maiores índices de internação ${ }^{14}$.

Em um estudo que incluiu 80 crianças com pneumonia comunitária internadas no Hospital da Restauração em Pernambuco, a prevalência também foi maior nas crianças do sexo masculino $(61,2 \%)^{15}$. Outra amostra, com 211 crianças indígenas hospitalizadas por infecção respiratória baixa, demonstrou maior frequência de hospitalização do sexo feminino ( $\mathrm{n}=109 ; 51,7 \%)$. Entretanto, o sexo masculino apresentou um maior acometimento por infecção de etiologia viral ${ }^{16}$.

Doenças do sistema respiratório, em sua maioria, afetam crianças de até 5 anos de idade, mas a faixa etária predominante está entre os 6 e os 24 meses $^{17}$. Essa foi a idade mais prevalente desta pesquisa, na qual $54,8 \%(n=34)$ das crianças tinham de 29 dias a 2 anos; e 27,4\% (n=17), de 3 a 6 anos de idade. Em um estudo recente, que descreveu o perfil sociodemográfico e epidemiológico de 669 crianças internadas em um hospital universitário ao norte do estado de Minas Gerais, 172 tiveram diagnósticos de doenças respiratórias. Destas, $122(70,9 \%)$ eram casos de pneumonia. Contudo, a maioria estava inserida na faixa etária de 1 a 4 anos (32,8\%), seguida da faixa entre 5 e 12 anos $(28,7 \%)$, menores de 6 meses $(27,9 \%)$ e de 6 a 12 meses $(10,6 \%)^{18}$.
Podemos constatar, em nossos resultados, com relação aos antecedentes pessoais, que pacientes com alguma doença preexistente do sistema neurológico compuseram uma significativa parcela $(n=11$; $18,0 \%)$, seguida da síndrome genética $(n=7 ; n=11,5 \%)$. Crianças com comprometimento neurológico podem apresentar insuficiência respiratória de origem central, refluxo gastroesofágico, tosse ineficaz, disfagia, disfunção oromotora e fraqueza da musculatura respiratória, sendo comumente hospitalizadas por pneumonia, incluindo pneumonias aspirativas ${ }^{19}$.

Nesta pesquisa, $16,1 \%(n=10)$ das crianças eram traqueostomizadas. A traqueostomia em crianças está associada a maiores riscos de complicações e taxas de morbidade e mortalidade ${ }^{20}$. Na prática clínica, tanto as crianças que apresentam comprometimento neurológico importante quanto as crianças traqueostomizadas, quando acometidas pela pneumonia, demandam mais cuidados por parte da equipe de fisioterapia, em decorrência de suas particularidades.

Quanto às complicações, o derrame pleural foi a mais frequente em nossa amostra, presente em $21,0 \%(n=13)$ das crianças. No que diz respeito à necessidade de tratamento em Unidade de Terapia Intensiva (UTI), o percentual foi de $20,3 \%(n=12)$. A necessidade de ventilação mecânica e ventilação não invasiva foi relativamente baixa $(1,7 \%)$ em ambas as modalidades. $\mathrm{Na}$ enfermaria do nosso serviço, essa demanda é composta por crianças que fazem uso desses suportes ventilatórios de maneira contínua. A oxigenoterapia foi necessária para 15 pacientes $(24,2 \%)$, e a interface mais utilizada foi o cateter nasal $(19,4 \% ; n=12)$.

A fisioterapia respiratória em crianças tem como propósito melhorar a função respiratória e reduzir os prejuízos causados pelas complicações pulmonares ${ }^{1}$. Em um estudo de revisão da literatura sobre evidências científicas de técnicas de fisioterapia respiratória convencionais e atuais em pediatria, os resultados foram controversos. Entretanto, verificou-se que é um recurso seguro, minimiza a gravidade do quadro clínico/disfuncional e não causa danos para a função respiratória ${ }^{21}$. Uma recente atualização do banco de dados Cochrane avaliou a eficácia da fisioterapia respiratória em crianças com pneumonia, concluindo que não houve indicação confiável sobre sua 
utilização. No entanto, esse resultado foi atribuído às discrepâncias entre os estudos e à apresentação estatísticas dos dados? .

Crianças hospitalizadas por doença respiratória aguda não devem ser submetidas rotineiramente às técnicas de higiene brônquica, pois sua indicação deve ser embasada em uma avaliação clínica individuali$z \operatorname{zada}^{22}$. Nesse contexto, ações que têm por objetivo o conhecimento das características de crianças hospitalizadas com pneumonia são importantes para nortear os protocolos de avaliação e conduta de maneira mais individualizada. Dessa forma, são necessários mais estudos com esse enfoque.

\section{CONCLUSÃO}

Verificou-se uma predominância do sexo masculino, com a faixa etária entre 29 dias e 2 anos; a complicação mais prevalente foi o derrame pleural; a oxigenoterapia foi necessária a um total de 15 dos 62 pacientes. Uma parcela significativa das crianças era traqueostomizada, ou tinha como antecedentes pessoais doenças neurológicas ou síndromes genéticas. Atualmente, a utilização da fisioterapia respiratória no tratamento de crianças com pneumonia é controversa, de modo que sua indicação e conduta devem ser embasadas na avalição clínica individualizada. O conhecimento de dados referentes às características dessas crianças possibilitará nortear protocolos que proporcionem uma melhor assistência.

\section{REFERÊNCIAS}

1. Pinto BF, Araújo PQ, Amaral JDF. Atuação da fisioterapia no esforço respiratório em crianças hospitalizadas com infecção respiratória aguda: um estudo comparativo. Fisioter Bras. 2017;18(2):140-7.

2. Sarmento GTV. Fisioterapia respiratória em pediatria e neonatologia. 2. ed. São Paulo: Manole; 2011. p. 36-60.
3. Das RR, Singh M, Naik SS. Vitamin D as an adjunct to antibiotics for the treatment of acute childhood pneumonia. Cochrane Database Syst Rev. 2018;7:CD011597.

4. McAllister DA, Liu L, Shi T, Chu Y, Reed C, Burrows J, et al. Global, regional, and national estimates of pneumonia morbidity and mortality in children younger than 5 years between 2000 and 2015: a systematic analysis. Lancet Glob Health. 2019;7(1):e47-e57.

5. Liu L, Oza S, Hogan D, Chu Y, Perin J, Zhu J, et al. Global, regional, and national causes of under-5 mortality in 2000-15: an updated systematic analysis with implications for the Sustainable Development Goals. Lancet. 2016;388(10063):3027-35.

6. Howie SRC, Murdoch DR. Global childhood pneumonia: the good news, the bad news, and the way ahead. Lancet Glob Health. 2019;7(1):e4-e5.

7. Murdoch DR, Howie SRC. The global burden of lower respiratory infections: making progress, but we need to do better. Lancet Infect Dis. 2018;18(11):1162-3.

8. Assunção RG, Pereira WA, Abreu AG. Pneumonia bacteriana: aspectos epidemiológicos, fisiopatologia e avanços no diagnóstico. Rev Investig Bioméd. 2018;10(1):83-92.

9. Balachandran A, Shivbalan S, Thangavelu S. Chest physiotherapy in pediatric practice. Indian Pediatr. 2005;42(6):559-68.

10. Corten L, Jelsma J, Human A, Rahim S, Morrow BM. Assisted autogenic drainage in infants and young children hospitalized with uncomplicated pneumonia, a pilot study. Physiother Res Int. 2017;23(1):1-8.

11. Parente JSM, Silva FRA. Perfil clínico-epidemiológico dos pacientes internados na clínica pediátrica em um hospital universitário. Rev Med UFC. 2017;57(1):10-4.

12. Maisel BA, Oliveira DA, Ferreira CAS, Righetti RF, Torquato JA, Cunha TMN, et al. Perfil epidemiológico das internações em uma unidade pediátrica do Sistema Único de Saúde. Fisioter Bras. 
2015;16(1):19-24.

13. Silva VLS, França GVA, Santos IS, Barros FC, Matiiasevich A. Characteristics and factors associated with hospitalization in early childhood: 2004 Pelotas (Brazil) birth cohort. Cad Saúde Pública. 2017;33(10):e00035716.

14. Araujo EMN, Costa GMC, Pedraza DF. Hospitalizations due to primary care-sensitive conditions among children under five years of age: cross-sectional study. Sao Paulo Med J. 2017;135(3):270-6.

15. Brito RCCM, Guerra TCM, Câmara LHLD, Mattos JDPG, Mello MJG, Correia JB, et al. Clinical characteristics and outcomes of acute community acquired pneumonia in children at a reference public hospital in Pernambuco State, Brazil (2010-2011). Rev Bras Saúde Matern Infant. 2016;16(3):247-57.

16. Souza PG, Cardoso AM, Sant'Anna CC, March MFBP. Acute lower respiratory infection in Guarani Indigenous children, Brazil. Rev Paul Pediatr. 2018;36(2):123-31.

17. Santos DSS, Santos MEF. Mortalidade por pneumonia em crianças menores de 5 anos no Brasil: Base de dados DATASUS. REBRASF Rev Bras Saúde Func. 2018;5(2):18-29.

18. Silva WB, Prado PF, Soares NM, Lima CA, Figueiredo ML, Oliveira VV. Crianças internadas em hospital universitário: caracterização sociodemográfica e epidemiológica. Rev Norte Min Enferm. 2017;6(1):18-31.

19. Thomson J, Hall M, Ambroggio L, Stone B, Srivastava R, Shah SS, et al. Aspiration and non-aspiration pneumonia in hospitalized children with neurologic impairment. Pediatrics. 2016;137(2):e20151612.

20. Schweiger C, Manica D, Becker CF, Abreu LSP, Manzini M, Sekine L, et al. Tracheostomy in children: a ten-year experience from a tertiary center in southern Brazil. Braz J Otorhinolaryngol. 2017;83(6):627-32.
21. Chaves GSS, Freitas DA, Santino TA, Nogueira PAMS, Fregonezi GAF, Mendonça KMPP. Chest physiotherapy for pneumonia in children. Cochrane Database Syst Rev. 2019;1:CD010277.

22. Morrow BM. Airway clearance therapy in acute paediatric respiratory illness: a state-of-the-art review. S Afr J Physiother. 2019;75(1):1295. 